\title{
Lucentis $\mathbb{R}^{\nabla}$ (ranibizumab) ABBREVIATED UK PRESCRIBING INFORMATION
}

Please refer to the SmPC before prescribing Lucentis $10 \mathrm{mg} / \mathrm{ml}$ solution for injection. Presentation: A glass singleuse vial containing $0.23 \mathrm{ml}$ solution containing $2.3 \mathrm{mg}$ of ranibizumab $(10 \mathrm{mg} / \mathrm{ml})$. Indications: The treatment of neovascular (wet) age-related macular degeneration (AMD). Administration and Dosage: Single-use vial for intravitreal use only. Lucentis must be administered by a qualified ophthalmologist experienced in intravitreal injections under aseptic conditions. The recommended dose is $0.5 \mathrm{mg}(0.05 \mathrm{ml})$. Treatment should be initiated with a loading phase of one injection per month for three consecutive months, followed by a maintenance phase in which patients should be monitored for visual acuity on a monthly basis. If the patient experiences a loss of greater than 5 letters in visual acuity (ETDRS or one Snellen line equivalent), Lucentis should be administered. The interval between two doses should not be shorter than 1 month. Before treatment, evaluate the patient's medical history for hypersensitivity. The patient should also be instructed to self-administer antimicrobial drops, four times daily for 3 days before and following each injection. Children and adolescents: Not recommended for use in children and adolescents due to a lack of data. Elderly: No dose adjustment is required. Consult SmPC for full administration details before using Lucentis. Contraindications: Hypersensitivity to the active substance or excipients. Patients with active or suspected ocular or periocular infections. Patients with active severe intraocular inflammation. Special warnings and precautions for use: Lucentis is for intravitreal injection only. Intravitreal injections have been associated with endophthalmitis, intraocular inflammation, rhegmatogenous retinal detachment, retinal tear and iatrogenic traumatic cataract. Monitor during week following injection for infections. Patients should be instructed to report symptoms suggestive of any of the above without delay. Increases in intraocular pressure have been seen within 1 hour of injection. Both intraocular pressure and perfusion of the optic nerve head should be monitored and managed appropriately. Concurrent use in both eyes has not been studied. Bilateral treatment at the same time could lead to an increased systemic exposure. There is a potential for immunogenicity and patients should report an increase in severity of intraocular inflammation. Lucentis has not been studied in patients who have previously received intravitreal injections. Lucentis should not be administered concurrently with other anti-VEGF agents (systemic or ocular). Withhold dose and do not resume treatment earlier than the next scheduled treatment in the event of the following: a decrease in best corrected visual acuity (BCVA) of $\geqslant 30$ letters compared with the last assessment of visual acuity; an intraocular pressure of $\geqslant 30 \mathrm{mmHg}$; a retinal break; a subretinal haemorrhage involving the centre of the fovea, or if the size of the haemorrhage is $\geqslant 50 \%$ of the total lesion area; performed or planned intraocular surgery within the previous or next 28 days. Discontinue treatment in cases of rhegmatogenous retinal detachment or stage 3 or 4 macular holes. Interactions: No formal interaction studies have been performed. Adjunctive use of verteporfin photodynamic therapy (PDT) and Lucentis in an open study showed an incidence of intraocular inflammation following initial combination treatment of $6.3 \%$ (2 of 32 patients). Pregnancy and lactation: Ranibizumab should not be used during pregnancy unless the expected benefit outweighs the potential risk to the foetus. Women of child-bearing potential should use effective contraception during treatment. Breast-feeding is not recommended during the use of Lucentis. Driving and using machines: The treatment procedure may induce temporary visual disturbances and patients who experience these signs must not drive or use machines until these disturbances subside. Undesirable effects: Serious adverse events related to the injection procedure included endophthalmitis, rhegmatogenous retinal detachment, retinal tear and iatrogenic traumatic cataract. Other serious ocular events included intraocular inflammation and increased intraocular pressure. There is a theoretical risk of arterial thromboembolic events (ATEs) following intravitreal use of VEGF inhibitors. A low rate of ATEs has been observed in the Lucentis clinical trials, with no difference between the treatment groups. Very common: Intraocular pressure increased, headache, vitritis, vitreous detachment, retinal haemorrhage, visual disturbance, eye pain, vitreous floaters, conjunctival haemorrhage, eye irritation, foreign body sensation in eyes, lacrimation increased, blepharitis, dry eye, ocular hyperaemia, eye pruritus, arthralgia, nasopharyngitis. Common: Anaemia, retinal degeneration, retinal disorder, retinal detachment, retinal tear, detachment of the retinal pigment epithelium, retinal pigment epithelium tear, visual acuity reduced, vitreous haemorrhage, vitreous disorder, uveitis, iritis, iridocyclitis, cataract, cataract subcapsular, posterior capsule opacification, punctuate keratitis, corneal abrasion, anterior chamber flare, vision blurred, injection site haemorrhage, eye haemorrhage, conjunctivitis, conjunctivitis allergic, eye discharge, photopsia, photophobia, ocular discomfort, eyelid oedema, eyelid pain, conjunctival hyperaemia, cough, nausea, allergic reactions, hypersensitivity, anxiety. Uncommon: Blindness, endophthalmitis, hypopyon, hyphaema, keratopathy, iris adhesion, corneal deposits, corneal oedema, corneal striae, injection site pain, injection site irritation, abnormal sensation in eye, eyelid irritation. Please refer to SmPC for full listing of all undesirable effects.

For UK: Adverse events should be reported. Reporting forms and information can be found at www.yellowcard.gov.uk. Adverse events should also be reported to Novartis Pharmaceuticals UK Ltd on (01276) 698370.

Legal category: POM, UK Basic NHS cost: £761.20 Marketing authorisation number: EU/1/06/374/001.

Marketing authorisation holder: Novartis Europharm Limited, Wimblehurst Road, Horsham, West Sussex, RH12 5AB, United Kingdom. Full prescribing information, including SmPC, is available from: Novartis Pharmaceuticals, Frimley Business Park, Frimley, Camberley, Surrey, GU16 7SR. Telephone: 01276 692255. Fax: 01276692508. 